\title{
NOTE ON THE MOTION OF FLUID IN A CURVED PIPE
}

\author{
W. R. Dean and J. M. Hurst
}

1. In the stream-line motion "of fluid in a curved pipe the primary motion along the line of the pipe is accompanied by a secondary motion in the plane of the cross-section. The secondary motion decreases the rate of flow produced by a given pressure gradient and causes an outward movement of the region where the primary motion is greatest. It is difficult to deduce these consequences from the exact equations of motion, but it is easy to do so if it is assumed that the actual secondary motion is replaced by a uniform stream; conditions in the central part of the section mainly determines the motion and here the secondary motion is approximately a uniform stream. The appropriate velocity of the stream can be determined from the relation that has been found experimentally between the rate of flow in a curved pipe and the pressure gradient.

2. It is assumed that the velocity components $(U, V, W)$ referred to cylindrical coordinates $(\varpi, \phi, z)$ are independent of $\phi$ and of the time $t$. The equations of motion and of continuity are then

$$
\begin{gathered}
U \frac{\partial U}{\partial \varpi}+W \frac{\partial U}{\partial z}-\frac{V^{2}}{\varpi}=-\frac{1}{\rho} \frac{\partial P}{\partial \varpi}+\nu\left(\frac{\partial^{2}}{\partial \varpi^{2}}+\frac{1}{\varpi} \frac{\partial}{\partial \varpi}-\frac{1}{\varpi^{2}}+\frac{\partial^{2}}{\partial z^{2}}\right) U, \\
U \frac{\partial V}{\partial \varpi}+W \frac{\partial V}{\partial z}+\frac{U V}{\varpi}=-\frac{1}{\rho \varpi} \frac{\partial P}{\partial \phi}+\nu\left(\frac{\partial^{2}}{\partial \varpi^{2}}+\frac{1}{\varpi} \frac{\partial}{\partial \varpi}-\frac{1}{\varpi^{2}}+\frac{\partial^{2}}{\partial z^{2}}\right) V, \\
U \frac{\partial W}{\partial \varpi}+W \frac{\partial W}{\partial z}=-\frac{1}{\rho} \frac{\partial P}{\partial z}+\nu\left(\frac{\partial^{2}}{\partial \varpi^{2}}+\frac{1}{\varpi} \frac{\partial}{\partial \varpi}+\frac{\partial^{2}}{\partial z^{2}}\right) W
\end{gathered}
$$

and

$$
\frac{\partial U}{\partial \varpi}+\frac{U}{\varpi}+\frac{\partial W}{\partial z}=0
$$

The boundary of the pipe is the surface obtained by rotating either a circle or a rectangle about the $z$ axis. In the first of these cases it is assumed that the radius $a$ of the circle is small compared with the radius $R$ of the curve traced out by the centre of the circle, and the corresponding assumption is made in the second case. The equations can now be simplified by replacing, for instance, the operator $\partial / \partial \varpi+1 / \varpi$ by $\partial / \partial \varpi$; and by replacing $\varpi$ by the constant $R$ in the term $V^{2} / \varpi$ in the first equation and in the pressure term in the second equation. This yields the approximate equations

$$
\begin{gathered}
U \frac{\partial U}{\partial \varpi}+W \frac{\partial U}{\partial z}-\frac{V^{2}}{R}=-\frac{1}{\rho} \frac{\partial P}{\partial \varpi}+\nu\left(\frac{\partial^{2}}{\partial \varpi^{2}}+\frac{\partial^{2}}{\partial z^{2}}\right) U, \\
U \frac{\partial V}{\partial \varpi}+W \frac{\partial V}{\partial z}=-\frac{1}{\rho R} \frac{\partial P}{\partial \phi}+\nu\left(\frac{\partial^{2}}{\partial \varpi^{2}}+\frac{\partial^{2}}{\partial z^{2}}\right) V, \\
{[\text { MATHEMATIKA } 6(1959), 77-85]}
\end{gathered}
$$




$$
U \frac{\partial W}{\partial \varpi}+W \frac{\partial W}{\partial z}=-\frac{1}{\rho} \frac{\partial P}{\partial z}+\nu\left(\frac{\partial^{2}}{\partial \varpi^{2}}+\frac{\partial^{2}}{\partial z^{2}}\right) W
$$

and

$$
\frac{\partial U}{\partial \varpi}+\frac{\partial W}{\partial z}=0
$$

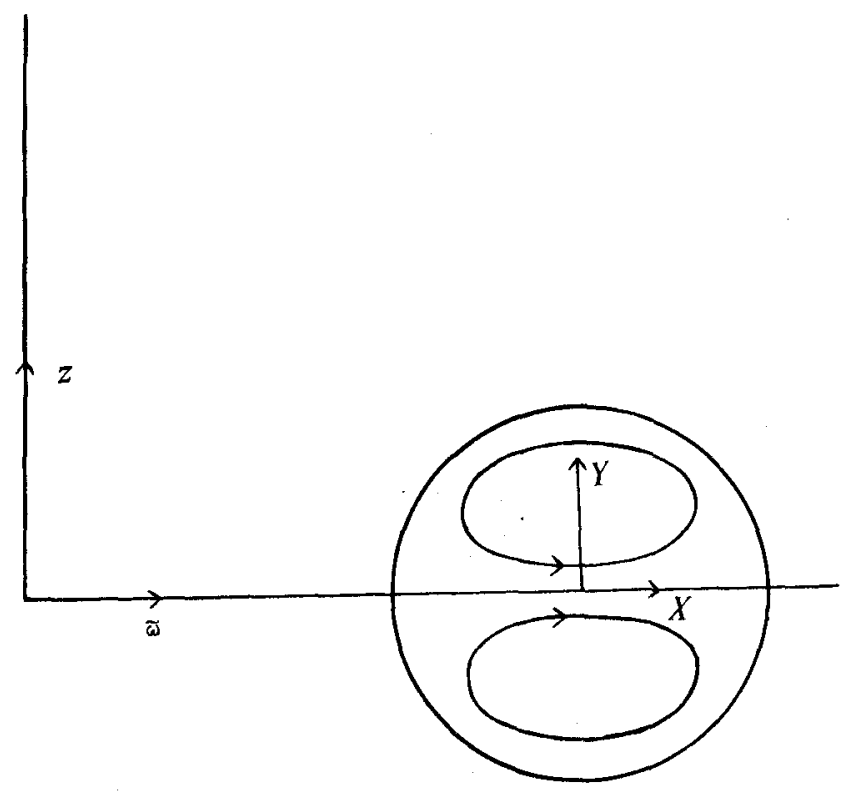

Fig. 1.

Equation (2) shows that the pressure $P$ must be of the form

$$
f(\varpi, z) \phi+g(\varpi, z)
$$

and it is then clear from equations (1) and (3) that $f(m, z)$ must be a constant. Hence

$$
P=-C R \phi+g(\varpi, z),
$$

where the constant $C$ is equal to the space-rate of decrease in pressure along the central line of the pipe. Equation (4) is satisfied if

$$
U=-\frac{\partial \Psi}{\partial z}, \quad W=\frac{\partial \Psi}{\partial \varpi}
$$

from equation (2)

$$
\nu\left(\frac{\partial^{2}}{\partial \varpi^{2}}+\frac{\partial^{2}}{\partial z^{2}}\right) V=-\frac{C}{\rho}+\left(\frac{\partial \Psi}{\partial \varpi} \frac{\partial}{\partial z}-\frac{\partial \Psi}{\partial z} \frac{\partial}{\partial \varpi}\right) V
$$

and the elimination of $P$ from (1) and (3) gives the equation

$$
\nu\left(\frac{\partial^{2}}{\partial \varpi^{2}}+\frac{\partial^{2}}{\partial z^{2}}\right)^{2} \Psi=\frac{2 V}{R} \frac{\partial V}{\partial z}+\left(\frac{\partial \Psi}{\partial \varpi} \frac{\partial}{\partial z}-\frac{\partial \Psi}{\partial z} \frac{\partial}{\partial \varpi}\right)\left(\frac{\partial^{2} \Psi}{\partial \varpi^{2}}+\frac{\partial^{2} \Psi}{\partial z^{2}}\right)
$$


3. In the approximate equations (7) and (8) the coordinates $m$ and $z$ appear only in the operators $\partial / \partial \varpi$ and $\partial / \partial z$; hence any point in the meridian plane that is selected for reference can be taken as the origin, and $X, Y$ can be used for the corresponding coordinates, Fig. 1. Non-dimensional forms of the equations in terms of $X, Y$ can now be obtained by writing

$$
\Psi=\nu \psi, \quad X=a x, \quad Y=a y, \quad V=V_{0} v,
$$

where $a$ and $V_{0}$ denote a length and a velocity such that

These equations are

$$
C a^{2}=4 \mu V_{0} \text {. }
$$

$$
\nabla_{1}^{2} v=-4+\left(\frac{\partial \psi}{\partial x} \frac{\partial}{\partial y}-\frac{\partial \psi}{\partial y} \frac{\partial}{\partial x}\right) v
$$

and

$$
\nabla_{1}^{4} \psi=K v \frac{\partial v}{\partial y}+\left(\frac{\partial \psi}{\partial x} \frac{\partial}{\partial y}-\frac{\partial \psi}{\partial y} \frac{\partial}{\partial x}\right) \nabla_{1}^{2} \psi
$$

where

$$
\nabla_{1}^{2} \equiv \frac{\partial^{2}}{\partial x^{2}}+\frac{\partial^{2}}{\partial y^{2}}
$$

and the non-dimensional constant $K$ is defined by

$$
K=\frac{2 a}{R}\left(\frac{a V_{0}}{\nu}\right)^{2} \text {. }
$$

If the pipe is straight, $K=0$; a solution of (11) is then $\psi=0$, and (10) becomes

$$
\nabla_{1}^{2} v=-4,
$$

which is the non-dimensional form of the known equation for the flow of liquid under pressure in a straight pipe. If the cross-section of the pipe is a circle of radius $a$, the appropriate solution of (13) is

$$
v=1-x^{2}-y^{2},
$$

and $V_{0}$ is the velocity of the fluid on the central line of the pipe. This defines $V_{0}$ as the velocity on the axis of a circular pipe of radius $a$ due to a pressure gradient $C$.

4. It is difficult to make much progress analytically with equations (10) and (11), but it is possible to deduce some of the effects of curvature from an approximate treatment. In equation (10) $\psi$ is the reduced stream function of the secondary motion, that is, of the motion in the plane of the cross-section which is roughly indicated in Fig. 1. The effect of the secondary motion is to reduce the total rate of flow through the pipe due to a given pressure gradient. Equation (10) may be regarded as determining the deflection of a flexible membrane by a normal pressure; it is then clear that the pressure in the central part of the membrane will normally have the most important effect on the deflection, and near the centre of the pipe the secondary motion is roughly in the $X$ direction, the velocity $V$ being 
right-handed about $O_{z}$. This suggests replacing the actual secondary motion by a uniform stream in the $X$ direction, i.e., writing

$$
\psi=-2 k y
$$

where $k$ is constant. The boundary conditions at the surface of the pipe will not, of course, be satisfied by the assumed secondary motion but the equation

$$
\frac{\partial^{2} v}{\partial x^{2}}+\frac{\partial^{2} v}{\partial y^{2}}=-4+2 k \frac{\partial v}{\partial x}
$$

that is derived from (10) by this approximation does, in fact, give in the two cases considered below a reduced total rate of flow.

5. Suppose first that the cross-section of the pipe is a circle of radius $a$, and let $x=r \cos \theta, y=r \sin \theta$ so that $r=1$ is the boundary of the section.

In (14) the substitution

leads to the equation

$$
v=\frac{2 x}{k}+u e^{k x}
$$

$$
\frac{\partial^{2} u}{\partial x^{2}}+\frac{\partial^{2} u}{\partial y^{2}}=\frac{\partial^{2} u}{\partial r^{2}}+\frac{1}{r} \frac{\partial u}{\partial r}+\frac{1}{r^{2}} \frac{\partial^{2} u}{\partial \theta^{2}}=k^{2} u
$$

The assumption that $u$ is proportional to $\cos n \theta$ gives Bessel's equation of order $n$ in $i k r$, and it can be seen that

$$
\frac{1}{2} k v=x+e^{k x} \sum_{n=0}^{\infty} \alpha_{n} I_{n}(k r) \cos n \theta
$$

where $\alpha_{0}, \alpha_{1}, \ldots$ are constants, is the solution of (14) that is required in this case.

The boundary condition, $v=0(r=1)$, is satisfied if

$$
\cos \theta e^{-k \cos \theta}=-\sum_{0}^{\infty} \alpha_{n} I_{n}(k) \cos n \theta,
$$

and it is known $([1], \mathrm{p} .36)$ that

$$
e^{-k \cos \theta}=I_{0}(k)+2 \sum_{1}^{\infty}(-)^{n} I_{n}(k) \cos n \theta
$$

whence $\quad \cos \theta e^{-k \cos \theta}=-I_{0}{ }^{\prime}(k)+2 \sum_{1}^{\infty}(-)^{n+1} I_{n}{ }^{\prime}(k) \cos n \theta$.

The boundary condition is accordingly satisfied if

$$
\alpha_{0}=I_{0}{ }^{\prime}(k) / I_{0}(k), \quad \alpha_{n}=2(-)^{n} I_{n}{ }^{\prime}(k) / I_{n}(k) \quad(n \geqslant 1) .
$$

The value of $v$ at the centre of the circle is

$$
2 k^{-1} I_{0}^{\prime}(k) / I_{0}(k)
$$

this is a decreasing function of $k$ with the correct value 1 when $k=0$. 
The total rate of flow through the pipe depends on $F_{1}$, the integral of $v$ taken over the area of the cross-section. From (15),

$$
F_{1}=\iint v d x d y=\frac{2}{k} \sum_{0}^{\infty} \alpha_{n} \int_{0}^{1} r I_{n}(k r)\left\{\int_{0}^{2 \pi} e^{k r \cos \theta} \cos n \theta d \theta\right\} d r .
$$

Since

$$
\int_{0}^{2 \pi} e^{k r \cos \theta} \cos n \theta d \theta=2 \pi I_{n}(k r) \quad(n \geqslant 0),
$$

and $([1]$, p. 70$)$

$$
\int_{0}^{1} r I_{n}^{2}(k r) d r=\frac{1}{2}\left(1+n^{2} k^{-2}\right) I_{n}^{2}(k)-\frac{1}{2} I_{n}^{\prime 2}(k) \quad(n \geqslant 0)
$$

it follows that

$$
\frac{k F_{1}}{4 \pi}=\frac{1}{2}\left[I_{0}(k)-\frac{I_{0}^{\prime 2}(k)}{I_{0}(k)}\right] I_{0}{ }^{\prime}(k)+\sum_{1}^{\infty}(-)^{n}\left[\left(1+\frac{n^{2}}{k^{2}}\right) I_{n}(k)-\frac{I_{n}^{\prime 2}(k)}{I_{n}(k)}\right] I_{n}{ }^{\prime}(k) .
$$

The integral $F_{1}$ is a decreasing function of $k$ with the correct value $\frac{1}{2} \pi$ when $k=0$.

6. It is now supposed that the cross-section is the rectangle bounded by the lines $X= \pm a, Y= \pm b= \pm \beta a$; then a solution of (14) is required such that $v=0$ when

$$
x= \pm 1,-\beta \leqslant y \leqslant \beta ; \quad y= \pm \beta,-1 \leqslant x \leqslant 1 .
$$

Let $\quad v=2\left(\beta^{2}-y^{2}\right)+e^{k x} \sum_{n=0}^{\infty}\left(A_{n} \cosh l_{n} x+B_{n} \sinh l_{n} x\right) \cos m_{n} y$,

where $A_{0}, A_{1}, \ldots, B_{0}, B_{1}, \ldots$ are constants and

$$
\beta m_{n}=\left(n+\frac{1}{2}\right) \pi, \quad l_{n}^{2}=k^{2}+m_{n}^{2} .
$$

Then equation (14) is satisfied, and $v=0$ if $y= \pm \beta$. The remaining conditions are satisfied if

$$
\left.\begin{array}{rl}
2\left(y^{2}-\beta^{2}\right) & =e^{k} \sum_{0}^{\infty}\left(A_{n} \cosh l_{n}+B_{n} \sinh l_{n}\right) \cos m_{n} y \\
& =e^{-k} \sum_{0}^{\infty}\left(A_{n} \cosh l_{n}-B_{n} \sinh l_{n}\right) \cos m_{n} y
\end{array}\right\}(-\beta \leqslant y \leqslant \beta) .
$$

Since

$$
y^{2}-\beta^{2}=\frac{4}{\beta} \sum_{0}^{\infty}(-)^{n+1} \frac{\cos m_{n} y}{m_{n}^{3}} \quad(-\beta \leqslant y \leqslant \beta),
$$

these conditions are satisfied if

$$
A_{n}=(-)^{n+1} \frac{8 \cosh k}{\beta m_{n}{ }^{3} \cosh l_{n}}, \quad B_{n}=(-)^{n} \frac{8 \sinh k}{\beta m_{n}{ }^{3} \sinh l_{n}},
$$

and this completes the determination of $v$, 
If $k=0, v$ can be found from a function $\Psi$ that gives the stress-distribution in the torsion of an elastic cylinder with a rectangular section [2]; $\Psi$ vanishes on the boundary and satisfies the equation $\nabla_{1}^{2} \Psi=-2$. From (17) and (18),

$$
v=2\left(\beta^{2}-y^{2}\right)+\frac{8}{\beta} \sum_{0}^{\infty}(-)^{n+1} \frac{\cosh m_{n} x \cos m_{n} y}{m_{n}^{3} \cosh m_{n}}
$$

if $k=0$, and it can be verified that (apart from a constant) this is in agreement with the formula given for $\Psi$.

It can be shown from (17) and (18) that

$$
\int_{-1}^{1} v d x=4\left(\beta^{2}-y^{2}\right)+\frac{8}{\beta} \sum_{0}^{\infty}(-)^{n+1} \frac{l_{n}\left(\cosh 2 l_{n}-\cosh 2 k\right)}{m_{n}^{5} \cosh l_{n} \sinh l_{n}} \cos m_{n} y,
$$

and hence that

If $k=0$,

$$
F_{2}=\iint v d x d y=\frac{16 \beta^{3}}{3}-\frac{16}{\beta} \sum_{0}^{\infty} \frac{l_{n}\left(\cosh 2 l_{n}-\cosh 2 k\right)}{m_{n}^{6} \cosh l_{n} \sinh l_{n}}
$$

$$
F_{2}=\frac{16 \beta^{3}}{3}-\frac{32}{\beta} \sum_{0}^{\infty} \frac{\tanh m_{n}}{m_{n}^{5}}
$$

7. Fig. 2 shows the effect of secondary motion in reducing the rate of flow. The upper curve shows the ratio of the rate of flow to the corresponding rate if $k=0$ for a pipe of circular section; the lower curve gives

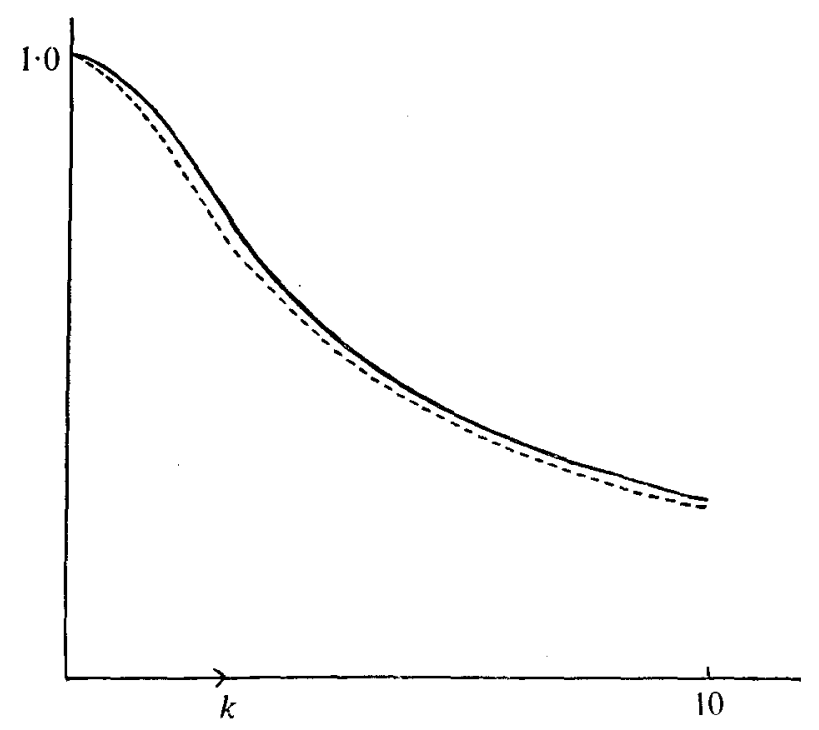

Fig. 2. Reduction in rate of flow

Circular section:

Square section : 
this ratio if the section is square. The reduction is greater in the latter case as would be expected, but the difference between the ratios is small throughout the range of values of $k$.

It is another consequence of curvature that the region where $V$, the velocity-component along the line of the pipe, takes its greatest values is moved outwards; this effect explains the cutting of a curved stream into the outer bank. This is shown in Fig. 3, again for pipes of circular and

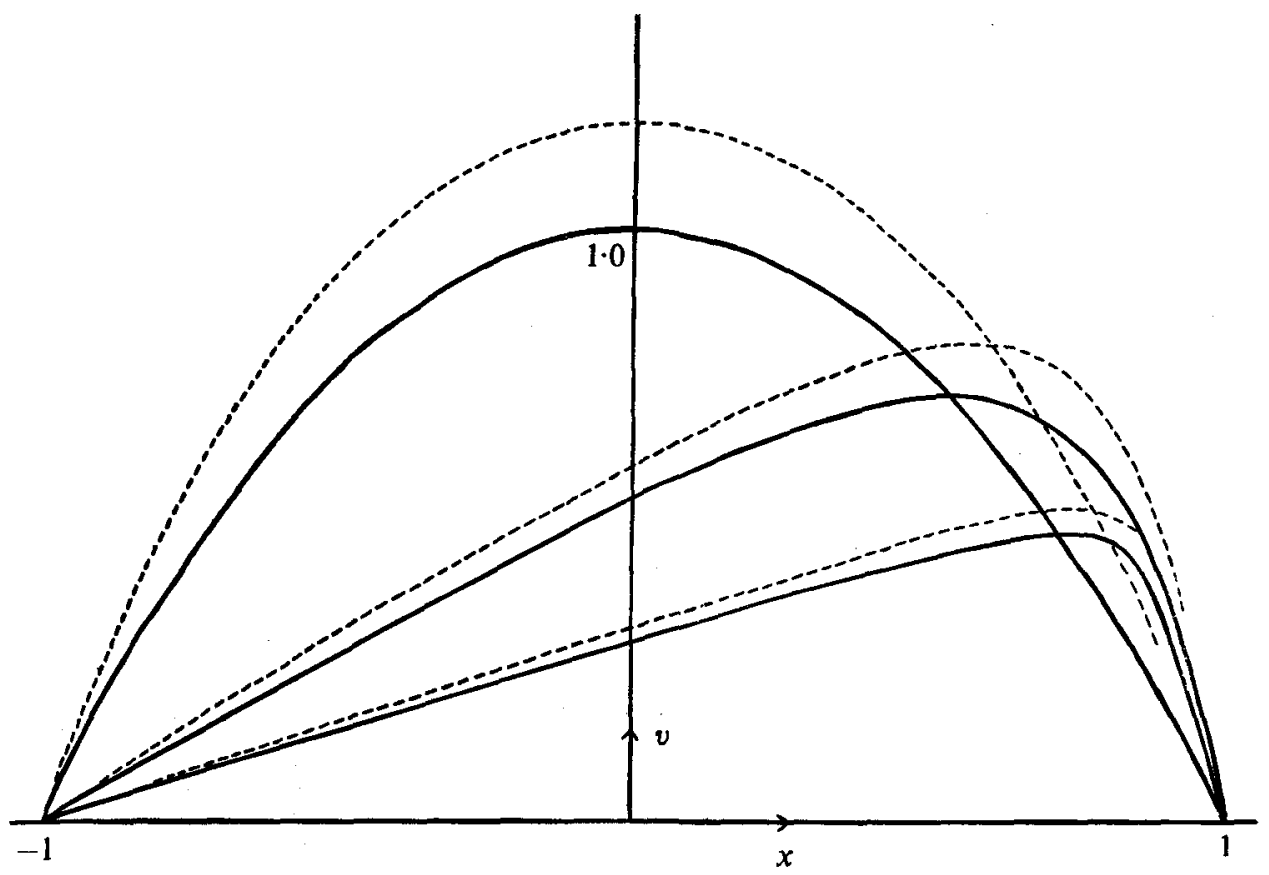

Fig. 3. Reduced velocity $v ; y=0 ; k=0,3,6$.

Circular section :

Square section :

square sections. The pairs of curves show the (reduced) velocity $v$ for values $-1 \leqslant x \leqslant 1, y=0$, and give the outward movement of the region of maximum velocity as $k$ increases.

Some calculations for rectangular sections with $\beta>1$ show an increase in both effects; this would be expected, since the effects should decrease as $\beta$ decreases, and in the limiting case $\beta \rightarrow 0$ curvature does not cause a secondary motion.

8. By experiment on the stream-line motion of fluid in curved pipes with circular sections, C. M. White [3] has found the relation between 
non-dimensional factors $\kappa$ and $C *$ defined by

$$
\kappa=\frac{2 a v_{m}}{\nu}\left(\frac{a}{R}\right)^{t}, \quad C^{*}=\left(\frac{\pi a^{4} C}{8 \mu}\right) /\left(\pi a^{2} v_{m}\right)
$$

$\pi a^{2} v_{m}$ and $C^{*} \pi a^{2} v_{m}$ are the rates of flow, under the same pressure gradient $C$, through the curved pipe and through a straight pipe with the same section. It follows that

$$
{ }_{\kappa} C^{*}=\left(\frac{a}{R}\right)^{\frac{1}{2}} \frac{C a^{3}}{4 \mu \nu} .
$$

Fig. 2 gives the relation, calculated from the assumed secondary motion, between $C^{*}$ and a variable $k ; k$ can therefore be regarded as a known function of $\kappa$ or $C^{*}$. If this is done the approximate theory can be used to estimate the ratio $U / V$ of the velocity-components at the centre of the pipe. At all points of the section $U=2 k \nu / a$, while at the centre

$$
V=\frac{2 I_{0}^{\prime}(k) V_{0}}{k I_{0}(k)}
$$

Hence at the centre

$$
\frac{U}{V}=\frac{k^{2} I_{0}(k)}{I_{0}{ }^{\prime}(k)} \cdot \frac{\nu}{a V_{0}}=\frac{k^{2} I_{0}(k)}{I_{0}{ }^{\prime}(k)} \cdot \frac{4 \mu \nu}{C a^{3}}
$$

or, in terms of $\kappa$ and $C^{*}$,

$$
\frac{U}{V}=\left(\frac{a}{R}\right)^{\frac{1}{2}} \frac{k^{2} I_{0}(k)}{I_{0}^{\prime}(k)} \cdot \frac{1}{\kappa C^{*}} .
$$

If $a / R$ is fixed, this ratio is a decreasing function of $k$ unless $k$ is small. From Fig. 2 the values of $C^{*}$ are $1 \cdot 24,2 \cdot 37$ and 3.56 for the values 2,6 and 10 of $k$, while from White's results (by interpolation) the corresponding values of $\kappa$ are 50,370 and 970 ; thus $k^{2} /\left(\kappa C^{*}\right)$ decreases from 0.065 to 0.029 as $k$ increases from 2 to 10 . The other factor $I_{0} / I_{0}{ }^{\prime}$ is a decreasing function of $k$ and tends to 1 as $k \rightarrow \infty$; it is $1 \cdot 43$ if $k=2$ and $1 \cdot 10$ if $k=6$.

The ratio $U / V$ is the angle that the stream-line passing through the centre of the circular section makes with the line of the pipe, and it can be shown theoretically that this angle at first increases with the rate of flow. The values calculated above suggest that the angle ultimately decreases, and for this there is some experimental evidence [4]. The calculations depend, of course, on the assumed form of the secondary motion, but the decrease in $U / V$ is considerable so that there is a margin for the error arising from the approximation. It is reasonable on physical grounds that the central value of $U / V$, a rough measure of the 
ratio of the secondary to the primary flow, should thus attain a maximum value.

\section{References.}

1. A. Gray and T. M. MacRobert, Bessel functions, 2nd ed. (London, 1922).

2. A. E. H. Love, Mathematical theory of elasticity, 4th ed. (Cambridge, 1934), p. 318.

3. C. M. White, Proc. Royal Soc., A, 123 (1929), 645-663.

4. J. Eustice, Proc. Royal Soc., A, 85 (1911), 122.

\section{University College,}

London, W.C.1. 